\title{
Stage IB Fallopian Tube Cancer AJCC v8
}

National Cancer Institute

\section{Source}

National Cancer Institute. Stage IB Fallopian Tube Cancer A/CC v8. NCI Thesaurus. Code C139986.

Stage IB includes: T1 b, N0, M0. T1 b: Fallopian tube cancer with tumor limited to fallopian tubes; no tumor on fallopian tube surface; no malignant cells in ascites or peritoneal washings. N0: No regional lymph node metastasis. MO: No distant metastasis. (AJCC 8th Ed.) 